\title{
Web-based Collaborative Platform for Personalized Orthopaedic Applications
}

\author{
Diana POPESCU ${ }^{1}$, Cătălin ILIE ${ }^{2}$, Dan LĂPTOIU ${ }^{3}$, Anton HADAR ${ }^{1}$, Raluca BARBUR ${ }^{2}$ \\ ${ }^{1}$ University POLITEHNICA of Bucharest, \\ 313, Splaiul Independentei, 060032, Bucharest 6, Romania, \\ diana@mix.mmi.pub.ro, anton.hadar@upb.ro \\ ${ }^{2}$ OSF Global Services, \\ 99, Bd. Dacia, 020053, Bucharest 2, Romania, \\ catalin.ilie@osf-global.com, raluca.barbur@osf-global.com \\ ${ }^{3}$ Colentina Clinical Hospital, \\ 19-21 Sos. Stefan cel Mare, 020125, Bucharest 2, Romania \\ dan.laptoiu@gmail.com
}

\begin{abstract}
The modern requirements of personalized medicine, the need of interdisciplinary approaches in developing medical devices and also the necessity to enhance communication between doctors and engineers impose the development of new e-health applications which can support different medical specialities or respond to patient individual needs. This is the context which helped determine current research to focus on the development of an intelligent online platform dedicated to design and manufacturing of patient-specific guides for orthopaedic surgery. The platform supports surgeonengineer collaboration and information sharing, offering online tools that allow inferring surgeon intents by using structured questionnaires adapted to the surgical intervention types and anatomical zones, and a collaborative 3D viewer for medical models and guides. Databases with medical and technical information, as well as with medical cases are implemented in the platform, being used for contextual information display and for information sharing and retrieval.
\end{abstract}

Keywords: web-based medical platform, collaborative design development, additive manufacturing, surgical guides

\section{Introduction}

Bridging the communication gap between doctors and engineers, enhancing the knowledge exchange and collaboration for developing personalized medical products are important objectives for both medicine and engineering [6], [7], [9] aiming at answering to the current growing demand of efficient and cost effective solutions for healthcare sector [15].

Personalized medicine means "providing the right treatment to the right patient at the right time" [4], one perspective of this approach being focused on designing, manufacturing and using customized instruments (such as surgical guides [3]) or implants [2] based on patient anatomical data.

In the same context, in the last couple of year, the number of e-health ICT systems and platforms increased significantly [14], all having the same general purposes: to improve the quality of care [10], to support medical decision [1], to enhance medical data visualization [13] or to remotely monitor patient health status [8].

Responding to these two trends: personalized medicine and e-health services, the research presented in this paper is focused on the development of a web-based platform for supporting the translation of orthopedic surgeons' requirements into technical specifications for designing and manufacturing patient' specific surgical guides (PSGs). Due to the fact these PSGs are customized to the patient anatomy they offer several important advantages: improvement of the accuracy of the surgical procedure, better orientation of the surgeon during intervention, decrease of the surgery time, risks of infections and costs.

PSGs match patient bones structures and are designed to contain geometrical features that materialize the pre-planned trajectory for a certain type of medical intervention (drilling, cutting). Their design (Figure 1) starts from

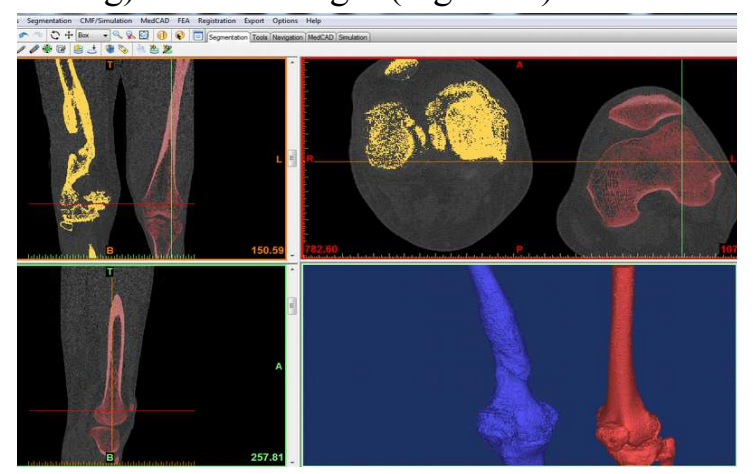

Figure 1. - a) 


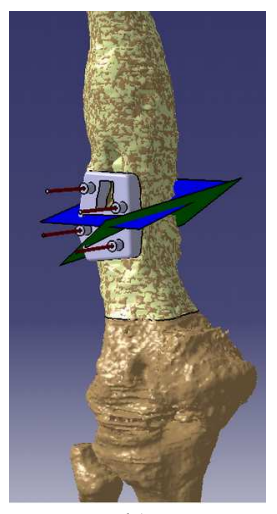

b)

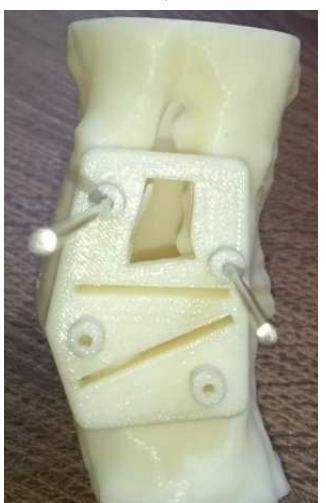

c)

Figure 1. Example of a patient specific guide and medical model:

a. medical modeling based on CT scanning data,

b. PSG design based on the reconstructed anatomical model,

c. physical replicas of virtual anatomical model and PSG patient Computer Tomography (CT) scanning data and involves: reconstructing the 3D model of the anatomical areas of interest and 3D modeling the surgical guide based on these areas, choosing the guide' material and manufacturing process, building the physical prototype of the personalized guide (usually employing an Additive Manufacturing - AM process), sterilization and, finally, use in the operation room. Thus, PSGs' design and manufacturing flow implies a tight surgeonengineer cooperation and information sharing for correctly establishing the correspondence between medical requirements and design, material and manufacturing issues.

This collaboration is mandatory for all the process steps, the use of intelligent decision support systems representing the key for overcoming the miscommunication problems.

Hence the idea to develop a web-based platform (called POIGO) that provides, in a collaborative environment, the necessary knowledge and computer-aided tools for supporting the exchange of medical and technical information between surgeon and engineer.

\section{POIGO Technical Specifications}

Data used by POIGO platform are:

1. Patient CT scans;

2. Medical knowledge (case studies, clinical studies, types of surgical guides presented in literature, information

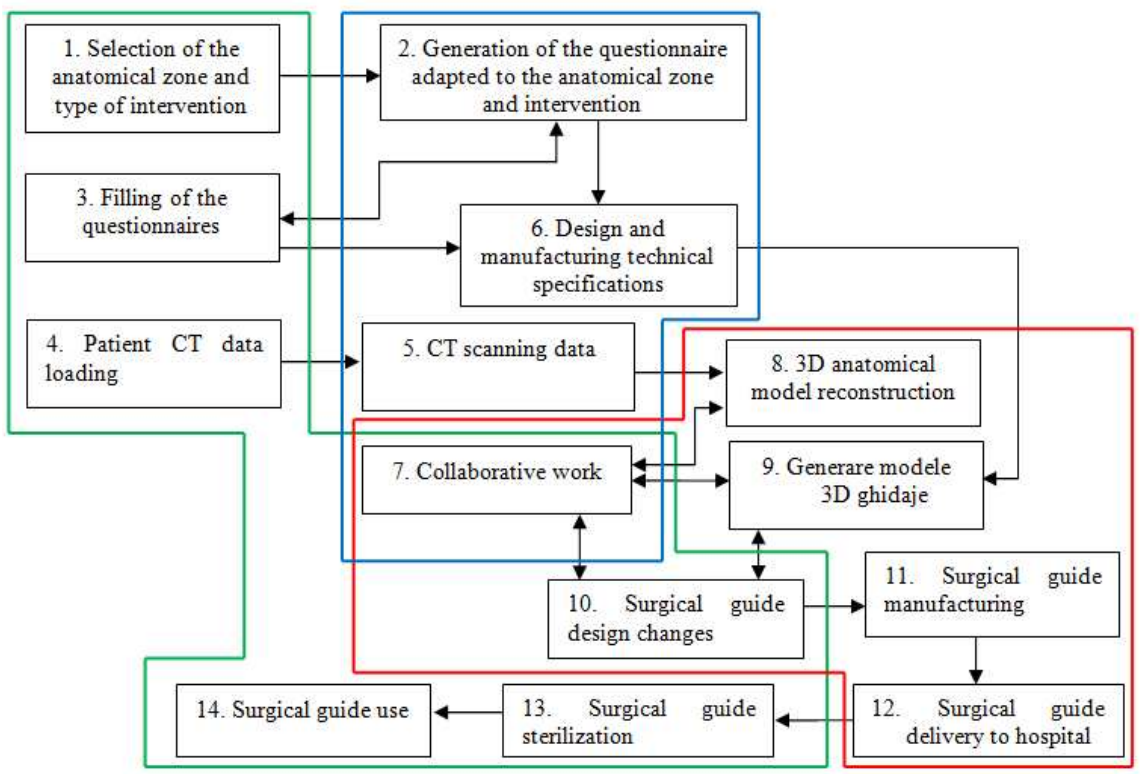

Figure 2. Design and manufacturing flow for PSGs using POIGO platform (green-surgeon tasks, blue-platform tasks, red-engineer tasks) 
specific to the type of surgical information, surgical tools and producers, etc.) incorporated in the database;

3. Technical knowledge incorporated in the database and used for contextual menus;

4. Medical information for a specific case, entered by the surgeon. These are input using two forms: structured questionnaires adapted to the surgical interventions for different anatomical areas (spine, foot, hip, hand, arm etc.) or narrative text input;

5. STL models for anatomical zones, PSGs;

6. Information provided collaboratively using direct selection of anatomical zones, points, drilling or cutting directions etc. on the $3 \mathrm{D}$ anatomical and guide STL models.

Platform output consists in reports generated based on surgeon input and sent to the engineer, transposing into a technical language different medical specifications, needs, constraints or requests. The physical result of the activities performed using POIGO platform is the surgical guide and the reconstructed anatomical model (both virtual and 3D printed).

In POIGO, the actions (details in [11]) are performed by surgeon, engineer, platform or in a collaborative manner, as can be seen from Figure 2. Therefore, POIGO accommodates several types of roles: administrator, surgeon, engineer and general user. Accordingly, for each role, access to applications, contextual information and database data are granted:

- Guest: general information on POIGO project and on PSGs advantages, case studies presented in literature, AM processes, manufacturing materials and sterilization methods, CT scanning protocol, etc.;

- Surgeon: project launching, choosing collaborator - engineer, questionnaires, CT data loading, 3D STL viewer and other communication tools;

- Engineer: technical specifications list, CT

- data, applications, 3D viewer and other communication tools;

- Administrator: users' accounts management, heterogeneous databases management, messages save management; reports generation, audits, etc.

The collaborative functionalities of the platform are achieved by integrating communication tools between surgeon and engineer needed to solve different situations, such as conflicting criteria, or to eliminate unfeasible designs from early stages.

These tools are specific to web communication, but include also a 3D STL viewer which offers the possibility to visualize the STL virtual models of patient anatomy and guides', to measure some geometrical features and to make annotation.

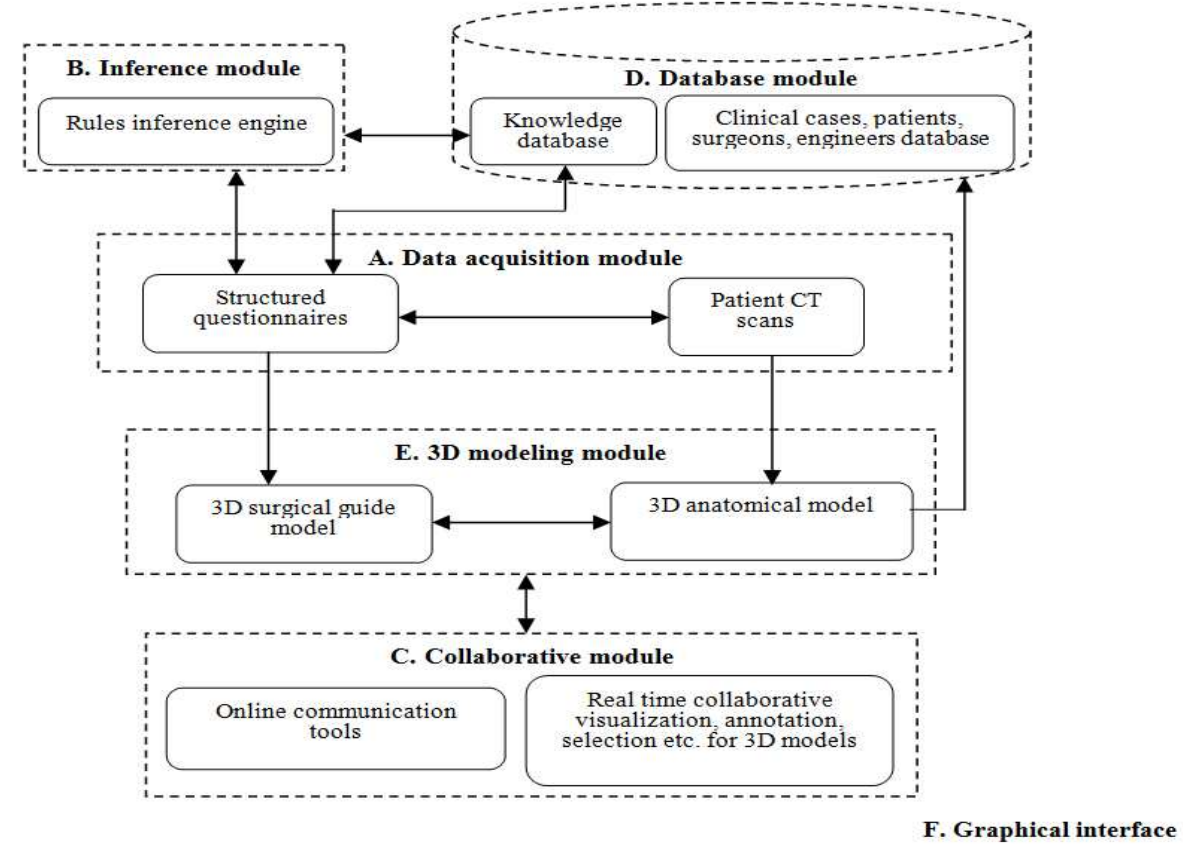

Figure 3. Schematic of POIGO platform: modules and data flow 
POIGO GUI (Graphical Users Interface):

- Uses data input and selection options like: dialog boxes, selection windows, contextual menus, text input fields;

- Uses standardized icons and buttons;

- Uses significant graphical images for supporting users' selection tasks;

- Includes options for interactively selecting points, measuring or annotating the $3 \mathrm{D}$ models displayed by the viewer.

Figure 3 presents the block scheme for POIGO, showing the modules included by the platform and data flow between them.

Questionnaires. In POIGO platform, the surgical needs are structured using different sets of questions adapted to the intervention type. The answers to these questionnaires are sent to the engineer as reports. Based on the cases presented in the literature [12] and on the project team members' experience, structured and adaptive questionnaires are set for the main type of interventions in which patient individualized surgical guides can be used. These constitute heuristics for the rules based inference engine.

Questionnaires should be available for the following anatomical zones:

- Spine - typical application: drill guides for inserting screws in the vertebra pedicles.

- Arm/Shoulder - typical application: osteotomy guides and drill guides for plates fixation using screws.

- Forearm/wrist/hand - typical application: osteotomy guides and drill guides for plates fixation using screws.

- Thigh/tibia/foot - typical application: osteotomy guides and drill guides for plates fixation using screws

- At the moment, questionnaire for Thigh/tibia/foot zone is implemented.

Contextual information. The contextual menu design is responsive. It adapts to the device which runs the application (for instance, monitors with different resolutions, mobile devices etc.), while the content is updated according to user' selections. The information offered depends on the current working object and ease the users' interaction with the application. Examples of such contextual info include, but are not limited to scientific papers (clinical studies, reviews, etc.); images presenting design of surgical guides presented in literature as case studies; CT scanning protocol, orthopedic products producers' catalogues (plates, screws, rods, sew blades, etc.).

Databases. In POIGO, two main types of databases are used: medical and technical knowledge database, and cases database. The first database provides information for the contextual menus, while the other database contains the lists of patients, cases, surgeons, engineers. Also, a CT scans archiving option can be activated by surgeon for attaching dicom files to a specific patient/case.

Viewer. A collaborative viewer should be also implemented in POIGO allowing:

- Real time collaborative visualisation of 3D STL models on surgeon and engineer computers, ipads or smart-phones;

- 3D STL models manipulation, measuring and annotation using points and lines;

- Selection of different anatomical zones from the 3D STL models for indicating the appropiate landmarks for PSGs' design.

Currently, platform' viewer functionality is under development.

\section{POIGO Platform Development}

\subsection{Questionnaires implementation}

The activity of structuring the questionnaires had started by defining the list of orthopedic surgical interventions which could benefit from the PSGs'. Literature analysis [12] served for defining the aforementioned list, but also the input and experience of orthopedic surgeons' from the research team was considered.

For questionnaires' implementation, structured series of questions and options for answers are defined, mentioning also the list of contextual information which should be available for user. For example, for designing an osteotomy guide for femur, the guide design will be the negative of selected supporting surfaces from the bone, but will also contain cutting slots for accommodating saw blade, as well as hollow cylinder for accommodated different diameters drill required for executing holes for $\mathrm{K}$-wires or screws (Figure 1.c). Other information is also available in the platform: list of references that present similar cases, guides' AM processes 
and corresponding materials, CT scanning protocol, sterilization protocol, models of fixation screws from different producers, etc.

The structured questionnaires are displayed the questions to follow this logical flow: Describe case $\rightarrow$ Choose application $\rightarrow$ Choose anatomical zone $\rightarrow$ CT data loading $\rightarrow$ Choose intervention type $\rightarrow$ Choose surgical guide type $\rightarrow$ Choose guide design $\rightarrow$ Select anatomical landmarks for design $\rightarrow$ Select cutting or drilling directions and entry points $\rightarrow$ Choose surgical tools (drills, saws).

In each displayed page/screen, the current selection step is visible for the surgeon at the top of the screen. More, in order to ease users' interaction with the application, a display of the summary of selections/options from the previous pages is available (Figure 4). Passing from a page to another is possible only after filling in the current page.

In every page of the application, Back functionality is implemented so that the user can return to previous pages and selections.

Further is presented an example of implemented pseudocode for a new case creation, questionnaire access and loading data in module A (Figure 3) of POIGO platform.

At the moment, in the platform are implemented the questionnaires for leg (Figures 5-6).

\section{New Case}

Add a new case in the system.

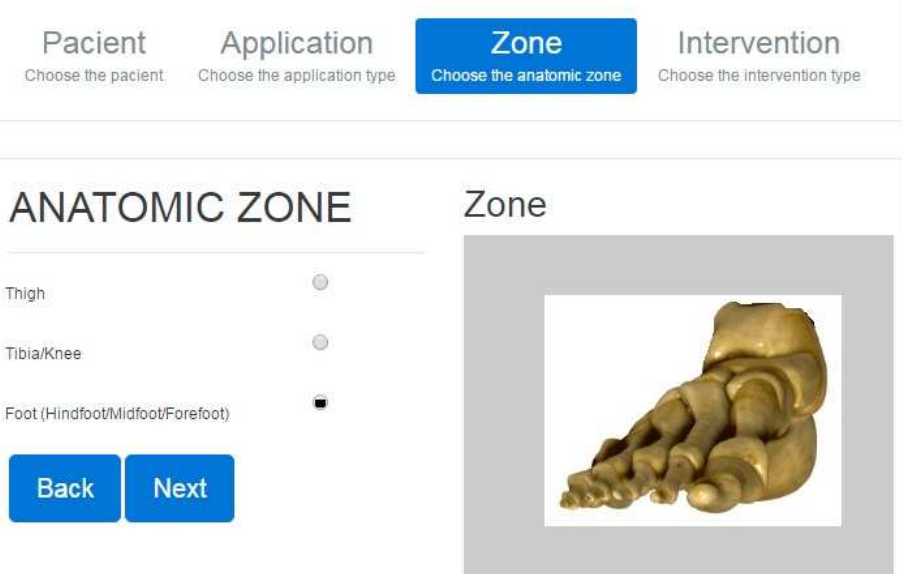

Figure 4. POIGO selection interface

\begin{tabular}{l} 
Contextual Content \\
$\square$ Help Articles \\
$\square$ Scientific Articles \\
$\square$ Case study \\
$\square$ Products \\
\hline
\end{tabular}

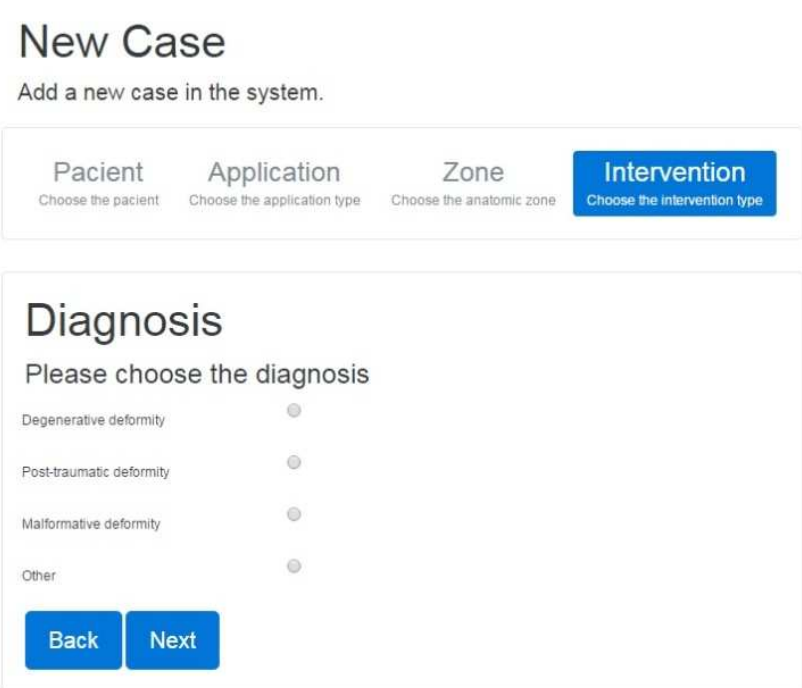

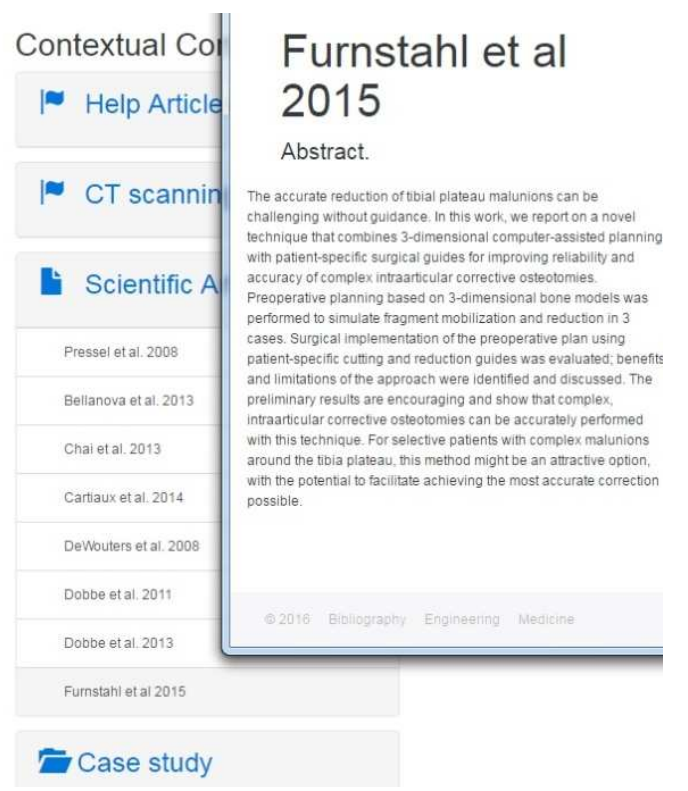

Figure 5. Screenshot from POIGO platform 


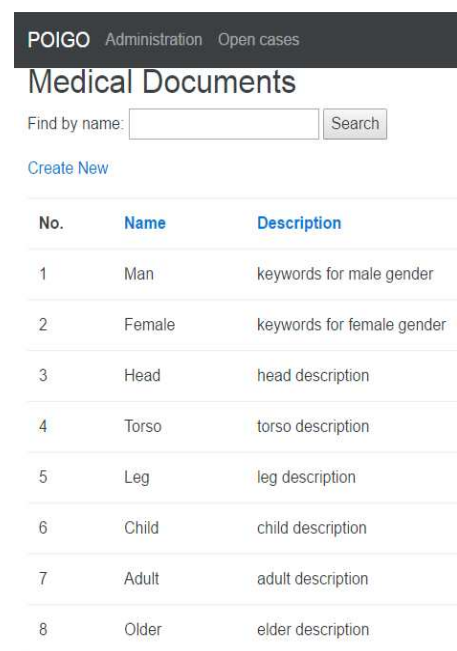

Page 1 of 1

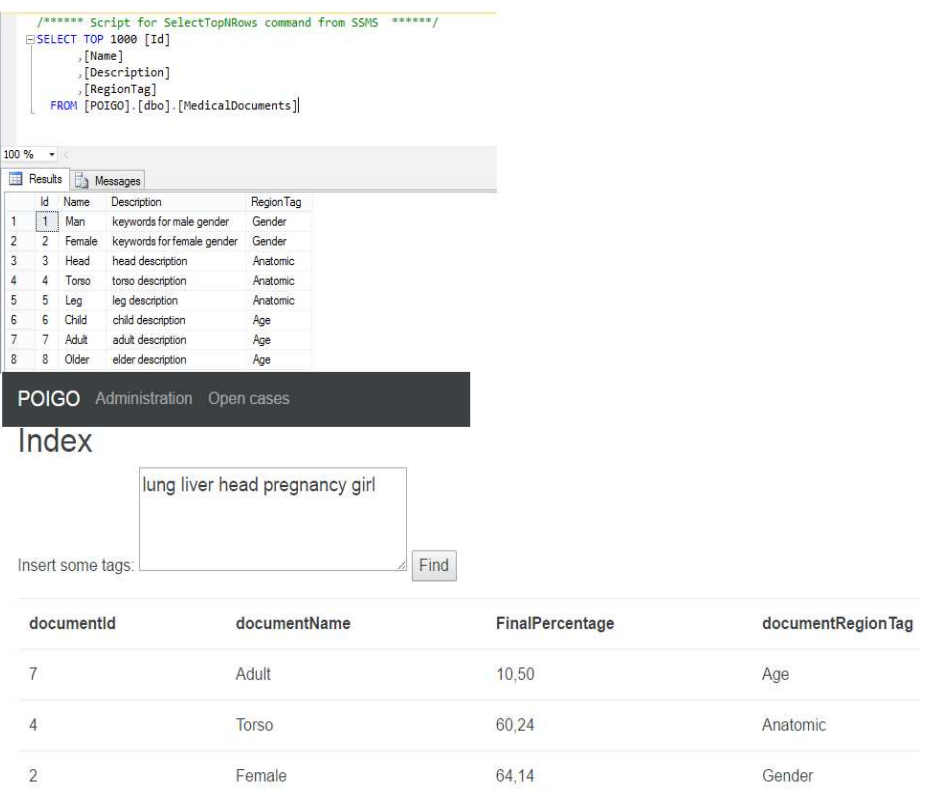

Figure 6. Data mining for medical case description

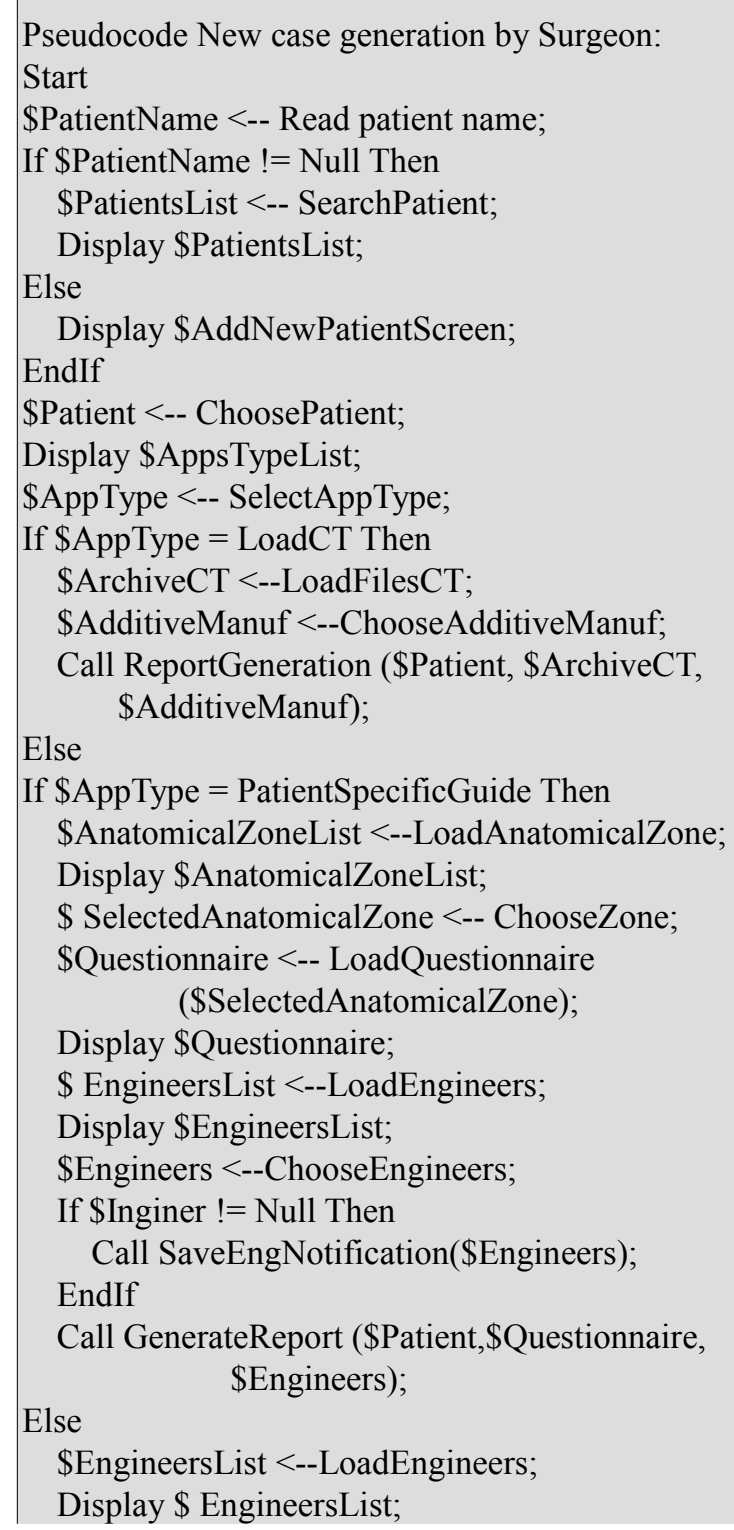

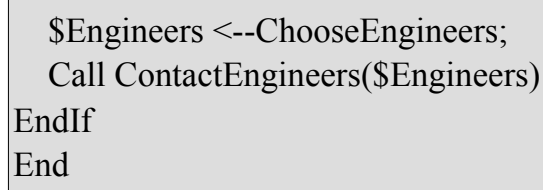

\subsection{Keywords extraction and recognition algorithm in POIGO}

As already mentioned, the initial information input by the surgeon can be narrative text (less than 300 characters) describing the case. This text is subjected to data mining methods and the following information is extracted: patient gender, age, anatomical zone of interest, diagnostic. This approach is appropriate because it can use unstructured text format, handle complex linguistic descriptions and vague concepts from specific terminology [5], which is the case with the information input by surgeon using free text for initial case description. Extracted data are used for sending, within the platform, brief alerts to registered engineers and also for filtering contextual medical information. Also, on the base of text mining analysis, automatic correlation with other cases from POIGO platform can be automatically inferred.

An example is presented further for clarifying the approach. When starting a new case for building a 3D anatomical model based on CT scans for a certain patient, the surgeon input a text describing the case: "Adult active male with valgus and recurvatum deformity of the femoral bone secondary to an old complicated 
fracture of the distal third of the right femur. Surgical guide and anatomical models needed for multi-planar osteotomy" or "Active male with chronic Charcot - type diabetic foot. CT X rays show bone remodeling with joint collapse and deformity of the right foot. Model for planning of bone resection (osteotomy, osteotomy) is needed for adequate bone reduction. Surgery for extended fusion (arthrodesis) of foot bones is required".

\subsubsection{TF-IDF algorithm (Term Frequency- Inverse Document Frequency).}

There are two stored procedures used for inserting and updating the knowledge base items for a specific medical document:

- UpdateStemRelevance;

- InsertIntoKnowledgeBase.

These procedures allow inserting keyword (stem of the word), document unique identifier, TFIDFvalue and Frequency parameters into database and computing the stem relevance parameter based on standard deviation algorithm.

When a medical document knowledge base is updated, if the same words are already into the implemented table, the frequency parameter is added and the highest TFIDF value is kept.

Tf-idf algorithm shows how significant a word is to a document, for this reason is being used in information retrieval and text mining as weight factor. This value modifies with the number of times a certain word appears in the document and with the frequency of the word in the document.

The algorithm was implemented in POIGO (Figure 6) to infer information from surgeon text input, as follows:

- Documents will be parsed and using a list of "stop-words" (words which are filtered out before processing of natural language data. Example: common and irrelevant words for our algorithm - the, in, it, etc.);

- Special characters, numbers and other useless characters are eliminated from documents;

- After documents standardization, the left words are considered keywords for our texts. Using TFIDF algorithm we are compute the TFIDFvalue and Frequency for each unique keyword.

\subsubsection{TFIDF-algorithm - principles and implementation:}

- If a word appears frequently in a document, then it is important and it will be rated with a high score;

- But if a word appears in many documents, then it is not a unique identifier and will get a low score.

Recognition algorithm. The following algorithm is applied for determining a document or a series of documents where the input tags belong:

a) Get the knowledge base items in lists of items based on stem relevance interval.

b) Compare our input tags (words) with every knowledge base item and flag found ones.

c) For each document, for each stem relevance interval are computed:

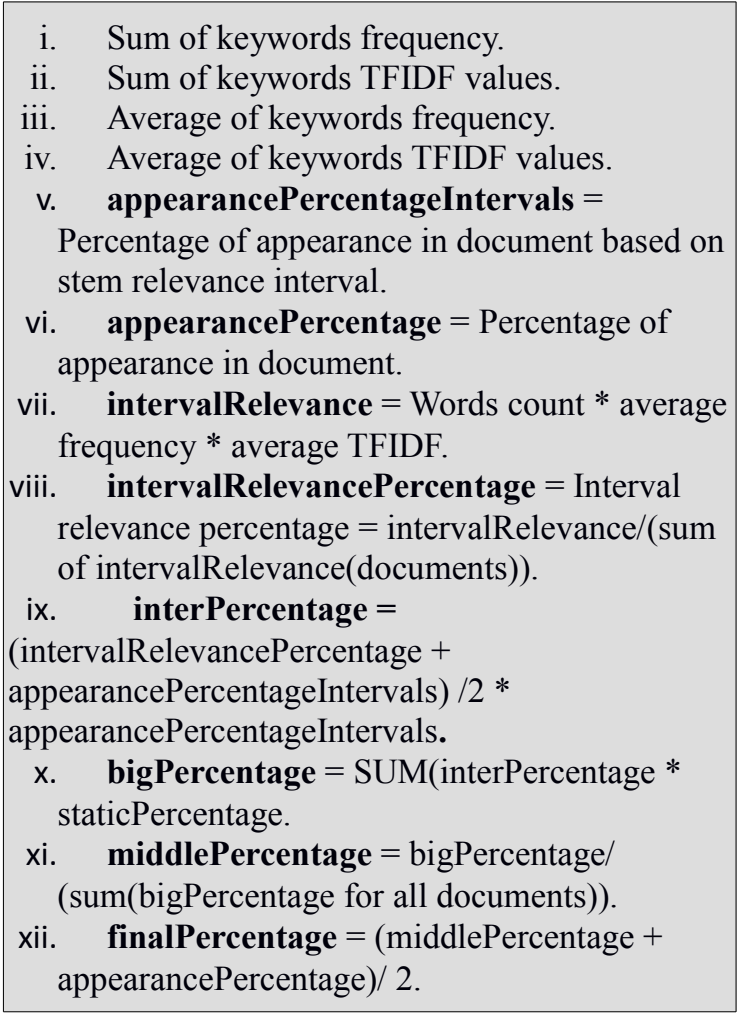

The finalPercentage parameter is the percentage of appearance of a series of words (input tags) into database knowledge base documents.

\subsection{POIGO rules based inference system}

POIGO platform implements rules for automating the conversion of medical requirements into design requirements. In this sense, questions and answers are used. When 
R1. If Anatomical zone $=$ Foot Then Display Optionslist $1=$ anatomical model, drill guide, other And Display ContextualMenu1

R2. If Selection=Drill guide Then Display Optionslist $2=$ one level guide, two levels guide, three levels guide And Display

ContextualMenu2

R3. If Selection= one level guide, Then Display Optionslist $3=$ cervical, thoracic, thoraco-lumbar, lumbar, lumbo-sacral, sacral And Display ContextualMenu3

R4. If Selection= lumbar Then Input value $=1 \ldots 5$ And Display Optionslist $4=$ negative surface design, V-shape design, other.

an answer is given to a question, the rule containing it is fired, and the action of further executing a succession of steps (other questions or actions) is activated. To each rule an action list is assigned. The action list contains the actions that are executed when the selected rule $(R)$ is fired. Further, examples of rules are presented for spine zone - drill guide design process.

Regarding contextual menus, as an example, ContextualMenu2 from above should contain bibliographic references on other drill guides, images with PSGs for one level of spine and for two and three spine levels, as well as similar cases developed using POIGO.

\subsection{Reporting functionality in POIGO}

The requirements, input by surgeons as answers to implemented questionnaires, are synthesized as reports and sent to the engineer.

There are three types of reports generated by POIGO, corresponding to three categories of outputs/results:

- Anatomical model - as 3D virtual model and as 3D printed physical model;

- Surgical guides based on CT patient data and 3D reconstructed model of the anatomical zone of interest;

\section{Report Details}

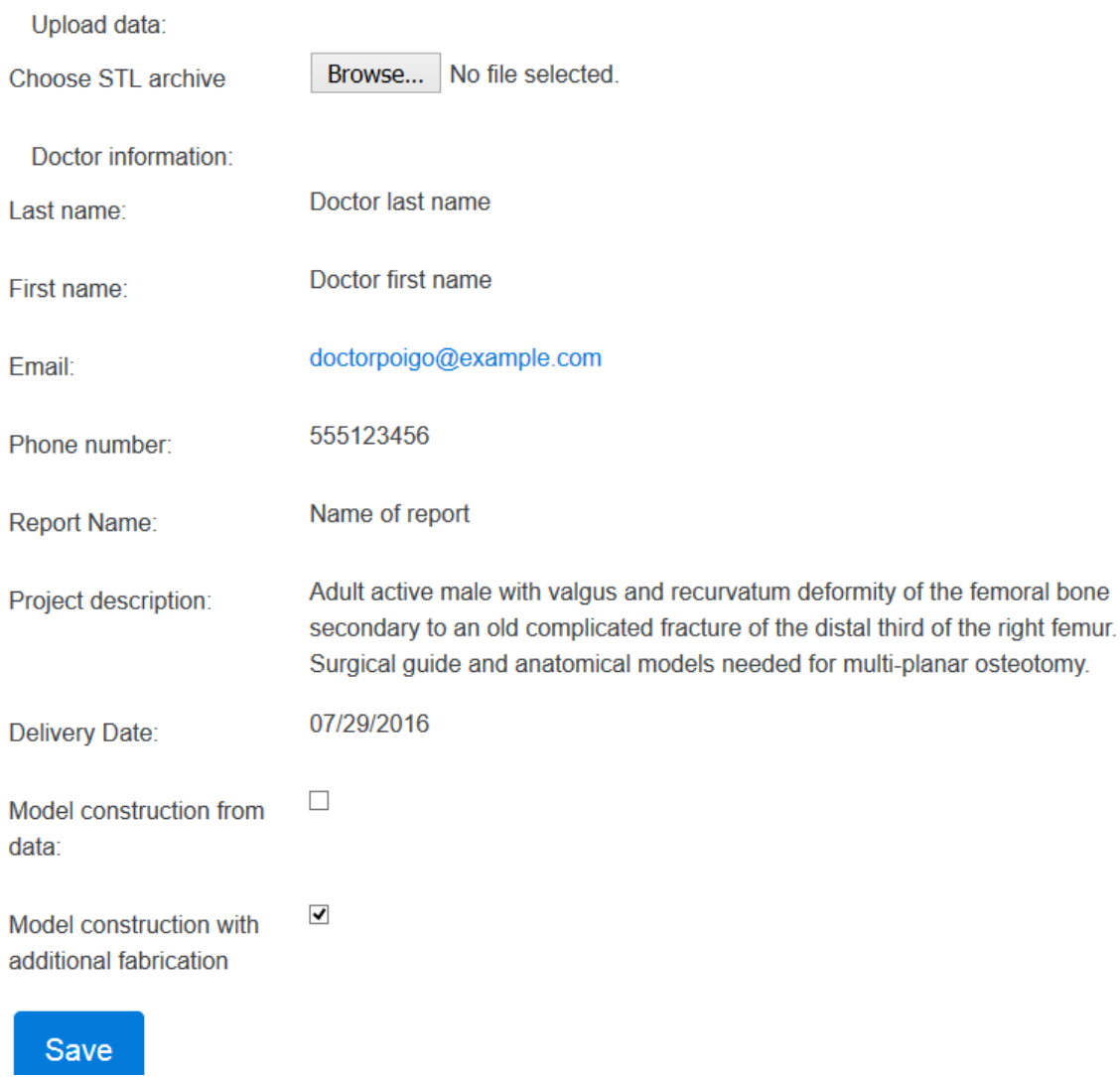

Figure 7. Example of a report automatically generated by POIGO platform 
- Other medical devices that should be patient customized and can be manufactured using AM processes.

These reports, an examples being presented in Figure 7, gathers together data input by surgeons and data deduced from surgeons' inputs, being presented as technical specifications.

\section{Conclusions and further work}

The advances in medical data visualization and processing, CAD software and AM technology paved the way towards the development of personalized surgical guides based on patient scanning data and used in certain procedure specific to orthopedic surgery. Our study shows how all these can be integrated in an online intelligent platform which allows a faster development of PSGs and enhances the collaboration between surgeon and engineer.

Structured questionnaires specific to the anatomical zone and type of interventions are used in the platform for quantifying medical requirements in terms of engineering design and manufacturing specifications. Also, a data mining algorithm for extracting information from the text input by surgeon is implemented as a tool for improving communication.

Currently, POIGO platform is in version POIGOLite, which means it offers only the functionality to reconstruct and manufactured $3 \mathrm{D}$ anatomical models based on patient $\mathrm{CT}$ data sent by surgeons. It also includes a $3 \mathrm{D}$ viewer with zoom and rotation options. The other functionality described are currently under testing by the project team members.

Further work will be focused on developing all the functionalities required for the 3D viewer and on implementing new structured questionnaires for other anatomical regions. Then, the work will be focused on piloting the platform for obtaining the final version. Real tests will be performed using the platform and feedback from users will be collected and analyzed.

\section{Acknowledgements}

The work has been funded by the Partnerships in Priority Areas Programme - PN II of MENUEFISCDI, through the Agreement 5/2014.

\section{REFERENCES}

1. ALMADANI, B. et al., E-Ambulance: Real-Time Integration Platform for Heterogeneous Medical Telemetry System, Procedia Computer Science, vol. 63, 2015, pp. 400-407.

2. ARORA, A., et al., Custom-Made Implant for Maxillofacial Defects Using Rapid Prototype Models, Journal of Oral Maxillo-facial Surgery, vol. 71(2), 2013, pp. e104-e110.

3. CARTIAUX, O., et al., Improved Accuracy with 3D Planning and Patientspecific Instruments during Simulated Pelvic Bone Tumor Surgery, Annual Biomedical Engineering, vol. 42(1), 2014, pp. 205-13.

4. ESF, Personalised Medicine for the European Citizen - Towards More Precise Medicine for the Diagnosis, Treatment and Prevention of Disease, http://www.esf.org/fileadmin/Public_docu ments/Publications/Personalised Medicine. pdf 2011, accessed February 2016.

5. GUPTA, V., et al., A Survey of Text Mining Techniques and Applications, Journal of Emerging Technologies in Web Intelligence, vol. 1(1), 2009, pp. 60-76.

6. HISARCIKLILAR, O. et al., Userdesigner Collaboration in the Design Process of Surgical Instruments: New Aspects for Annotation as a Communication Tool, Conference ICED'09, Stanford University, 2009, pp. 1-11.

7. LOOI, T., Interface between Engineering and Medicine, Chapter 1, Bio-engineering for Surgery, Critical Engineer Surgeon Interface, 2016, pp. 1-16.

8. PAWAR, P., et al., A Framework for the Comparison of Mobile Patient Monitoring Systems, Journal of Biomedical Informatics, vol. 45(3), 2012, pp. 544-556.

9. PAGLIARI, C., Design and Evaluation in ehealth: Challenges and Implications for an Interdisciplinary Field, Journal of Medical Internet Research. Vol. 9, iss. 2, 2007, pp. e15. 
10. POP, R. M., et al., A Web-based Nutritional Assessment Tool, Studies in Informatics and Control journal, vol. 22(3), 2013.

11. POPESCU, D., et al. Workflow for Additive Manufacturing of an Individualized Surgical Template, Journal of Proceedings in Manufacturing Systems, ISSN 2067-9238, vol. 10(3), 2015, pp. 131-140.

12. POPESCU, D., D. LĂPTOIU, Rapid Prototyping for Patient-specific Surgical Orthopedics Guides: A Systematic Literature Review, vol. 230, iss. 6, 2016, pp. 495-515.

13. SHNEIDERMAN, B., et al, Improving Health and Healthcare with Interactive
Visualization Methods, IEEE Computer, Special Issue on Challenges in Information Visualization, vol. 46(5), 2013, pp. 58-66.

14. TIEMAN, J., S. L. BRADLEY, Systematic Review of the Types of Methods and Approaches Used to Assess the Effectiveness of Healthcare Information Websites, Australian Journal of Primary Health, vol. 19(4), 2013, pp. 319-324.

15. TORBETT, R., Cost-Efficiency and the Road to Investment, European Alliance for Personalised Medicine, http://euapm.eu/pdf/EAPM_Cost_Efficienc y_and_Road_to_Investment.pdf 2014, accessed February 2016. 